\title{
Incidental benefits after fecal microbiota transplant for ulcerative colitis
}

\author{
Ramit Mahajan ${ }^{1}$, Vandana Midha ${ }^{2}$, Arshdeep Singh ${ }^{1}$, Varun Mehta ${ }^{1}$, Yogesh Gupta ${ }^{1}$, Kirandeep Kaur ${ }^{3}$, \\ Ritu Sudhakar ${ }^{4}$, Anmol Singh Pannu ${ }^{1}$, Dharmatma Singh ${ }^{1}$, Ajit Sood ${ }^{1}$ \\ Departments of ${ }^{1}$ Gastroenterology, ${ }^{2}$ Internal Medicine, ${ }^{3}$ Pharmacology, and ${ }^{4}$ Dietetics, Dayanand Medical College and Hospital, Ludhiana, \\ India
}

Gut dysbiosis can result in several diseases, including infections (Clostridium difficile infection and infectious gastroenteritis), autoimmune diseases (inflammatory bowel disease, diabetes, and allergic disorders), behavioral disorders and other conditions like metabolic syndrome and functional gastrointestinal disorders. Amongst various therapies targeting gut microbiome, fecal microbiota transplantation (FMT) is becoming a focus in the public media and peer reviewed literature. We have been using FMT for induction of remission in patients with moderate to severe active ulcerative colitis (UC) and also for subsequent maintenance of remission. Four cases reported incidental benefits while being treated with FMT for UC. These included weight loss $(\mathrm{n}=1)$, improvement in hair loss $(\mathrm{n}=1)$, amelioration of axial arthritis $(\mathrm{n}=1)$ and improvement in allergic rhinitis $(\mathrm{n}=1)$, thereby suggesting potential clinical applications of FMT in treating extraintestinal diseases associated with gut dysbiosis. (Intest Res 2020;18:337-340)

Key Words: Fecal microbiota transplantation; Colitis, ulcerative; Incidental findings

\section{INTRODUCTION}

Fecal microbiota transplantation (FMT) has been shown to be effective in active UC by targeting gut dysbiosis. We have been using FMT for induction of remission in patients with moderate to severe active UC and also for subsequent maintenance of remission. ${ }^{1,2}$ In our center, fecal slurry made from freshly passed stools from a screened donor is administered into the terminal ileum by a colonoscopic route, in a prepared colon. The protocol for FMT involves 7 sessions; at weeks 0 , 2, $6,10,14,18$, and 22 for induction of remission followed by 8 weekly FMT infusions for maintenance of remission. ${ }^{1,2}$ A pro-

Received September 8, 2019. Revised November 27, 2019.

Accepted December 20, 2019.

Correspondence to Ajit Sood, Department of Gastroenterology, Dayanand

Medical College and Hospital, Tagore Nagar, Ludhiana 141001, Punjab, India.

Tel: +919815400718, Fax:+911612302620,E-mail: ajitsood10@gmail.com spectively maintained electronic database of all these patients is reviewed regularly for clinical outcomes and status of co-morbid conditions, if any. Four patients noticed improvement in their co-morbid conditions while being on FMT for UC, without any specific treatment being offered for such diseases. We hereby report these incidentally reported benefits with FMT. These cases were incidentally detected during a study on role of FMT in patients with UC, which was approved by the Institutional Review Board (IEC No. 2015-113). Written consent was obtained from all patients.

\section{CASE REPORTS}

\section{Case 1}

A 24-year-old female patient had UC (E3 disease) for 2 years. Her disease was severe (Mayo score 11) and steroid refractory. She had received 3 doses of infliximab for induction of re- 
mission, and was maintained on mesalamine and azathioprine. However, she had a relapse 3 months later, which was managed with intravenous steroids. After an initial clinical response with intravenous steroids, FMT was started in addition to oral steroids and azathioprine. After 7 sessions of FMT she achieved clinical remission. She was obese before starting FMT (weight $65 \mathrm{~kg}$, BMI $28.0 \mathrm{~kg} / \mathrm{m}^{2}$ ), and her weight had been the same for the past 5 years. While receiving FMT, she started noticing loosening of clothes after third session and reported losing $12 \mathrm{~kg}$ at the end of 7 sessions (post-FMT: weight $53 \mathrm{~kg}$, BMI $23.5 \mathrm{~kg} / \mathrm{m}^{2}$ ). A diet diary maintained during the period she received FMT did not reveal any major changes in the type of food she consumed or the total caloric intake per day.

\section{Case 2}

A 34-year-old female patient had moderately active UC for 6 years. She also reported significant hair loss for which she had used multivitamins and topical agents (minoxidil), but there was no sustained benefit. During a relapse 1 year ago, she was treated with FMT after a partial response to steroids (azathioprine was not added). After 7 sessions of FMT, she achieved clinical remission. In addition to this, she also reported a significant improvement in hair loss. The number of hair on her hairbrush after combing, or that noted in the drain after shampooing had significantly decreased and she perceived an increase in the hair growth (according to the 4-item Women's Hair Growth Questionnaire). She denied concomitant use of any drugs (oral or topical) to enhance hair growth. A traction test was done and Sabouraud sign was negative. She has been on follow up for 6 months and has a sustained improvement in hair loss.

\section{Case 3}

A 38-year-old male patient had moderately active (Mayo clinic score 8) steroid dependent UC. He had inflammatory back pain for more than 1 year, which was insidious in onset, associated with morning stiffness and improvement with physical exercise. Radiographs of the lumbosacral spine and sacroiliac joints were normal. Initial treatment with sulfasalazine had to be stopped due to GI intolerance, and he was on mesalamine and corticosteroids when FMT was started. After 7 sessions of FMT, he achieved clinical remission and corticosteroids were gradually tapered off. He also reported relief in his back pain and reduction in morning stiffness. Over the next 6 months of follow up, he did not require corticosteroids or NSAIDs for analgesia.

\section{Case 4}

A 29-year-old female patient had moderately active steroid dependent UC of 2 years duration. She also had recurrent episodes of rhinorrhea, itching and nasal congestion since childhood for which she had been on several anti-histaminic drugs. After 7 sessions of FMT, she achieved clinical remission and could withdraw corticosteroids. Despite this, she reported resolution of her symptoms of allergic rhinitis and could discontinue anti-histaminic drugs.

\section{DISCUSSION}

UC is a chronic multifactorial disease that affects the GI tract and results from an abnormal immune response to the luminal antigens in genetically predisposed individuals. Gut dysbiosis plays an important role in pathogenesis of UC, in which a reduction in anti-inflammatory phyla, Firmicutes and Bacteroidetes and an increase in pro-inflammatory Proteobacteria such as Enterobacteriaceae has been reported. Several other diseases also result due to gut dysbiosis, including infections (Clostridium difficile infection and infectious gastroenteritis), autoimmune diseases (diabetes and allergic disorders), behavioral disorders and other conditions like metabolic syndrome and functional GI disorders. ${ }^{3}$ Some of these co-morbid immune mediated disorders may simultaneously be present in patients with UC. Though recent studies have proven the efficacy of FMT in induction and maintenance of remission in UC, its effect on the course of other immune mediated disorders is yet unclear. However, when treated separately, efficacy of FMT in some of these conditions has been reported.

Case no. 1 reported significant, sustained weight loss. The prevalence of obesity in IBD has risen in parallel with the general population. Pro-inflammatory adipokines produced by adipose tissue, environmental risk factors, and alterations in the gut microbiome may explain epidemiologic links between obesity and IBD. ${ }^{4}$ Obesity also negatively influences IBD disease course (risk of complication, surgery, hospitalization, and infections) and response to medical therapy (including biologics). ${ }^{5}$ Weight loss, as seen in patient $\mathrm{A}$, can be attributed to changes brought about in the intestinal milieu by a changed microbiome post-FMT. Environmental factors associated with both obesity and IBD such as diet and exposure to antibiotics are likely modulated through gut microbiome. Targeting obesity via manipulation of intestinal microbiome may be the future therapeutic approach to obesity in general population and IBD in particular. 
Hair fall, a common clinical problem in IBD, has been reported only anecdotally. The causes for disease related hail-fall include telogen effluvium, drug side effects and nutritional deficiencies among others. Alopecia areata, an autoimmune disorder shares genetic risk factors with IBD and both these disorders have altered immune response, characterized by increased levels of interferon- $\gamma$ and TNF- $\alpha .{ }^{6}$ In addition to this, luminal metabolic alterations due to gut dysbiosis and dietary modifications have been shown to alter skin physiology and result in alopecia. ${ }^{7}$ FMT corrects gut dysbiosis which may result in reduction of these pro-inflammatory cytokines and correction of luminal metabolic alterations resulting in improvement in gut inflammation and hair fall. Improvement in the nutritional status of the patients after achieving remission may also reduce the hair loss. Recent case reports of hair growth noted in 2 patients with alopecia areata when treated with FMT for recurrent $C$. difficile infection suggest the same. ${ }^{8}$

Arthritis is a common extraintestinal manifestation of IBD, occurring in approximately $30 \%$ of IBD patients and significantly affecting quality of life. Oligoarticular peripheral arthritis parallels the disease activity of IBD, hence treatment of IBD may resolve this type of arthritis. Polyarticular and axial arthritis however, are independent of disease activity, and require specific treatment. Recent studies have suggested a causal relationship between the intestinal microbiota and spondyloarthritis and thus linking dysbiosis of the complex microbial communities with pathogenesis of spondyloarthritis. Animal models reveal that a germ-free environment is preventive for development of gut and joint disease, suggesting that microbial exposure is necessary for arthritis to develop in the presence of predisposing genetic background. ${ }^{9}$ Gut dysbiosis may alter immune balance in the lamina propria towards a pro-inflammatory state, with an increased interleukin (IL)-23 production and activation of $\gamma / \delta$ T cells capable of amplifying the response through cytokines such as IL-17 and TNF, resulting in both gut and joint inflammation. ${ }^{10}$ A classic example of altering gut microbiome for therapeutic benefits in inflammatory arthritis is sulfasalazine, which is a combination of antibacterial sulfonamide bonded with a salicylate through an azo bond. However, this drug has several adverse events restricting its use. Correction of gut dysbiosis with FMT may improve both IBD and associated inflammatory arthritis, and no major adverse events have been reported with the same so far. Larger studies with detailed microbiome analysis can be considered based on this observation.

Two determinants for development allergic disorders have been proposed; hygiene hypothesis and disruption of intestinal microbiota. Animal models and longitudinal human studies reverberate the relationship between allergic diseases and microbiota. ${ }^{11}$ The use of FMT may restore immune homeostasis by enabling colonization of a sturdier and resilient community of microbes thereby alleviating symptoms attributable to allergic disorders.

To conclude, FMT seems to have potential clinical applications in treating a wide spectrum of extraintestinal diseases associated with gut dysbiosis. Correction of gut dysbiosis with FMT not only results in mucosal healing in the gut, but also has profound systemic, immunological and metabolic response. However, few questions remain unanswered. Will they respond to the same protocol, same route of administration and same dose of FMT? Will the response be similar? Can they relapse when FMT is stopped? Larger studies with evaluation of microbiome changes are needed to answer these questions.

\section{FINANCIAL SUPPORT}

The authors received no financial support for the research, authorship, and/or publication of this article.

\section{CONFLICT OF INTEREST}

No potential conflict of interest relevant to this article was reported.

\section{AUTHOR CONTRIBUTION}

Conception and design: Mahajan R, Midha V, Sood A. Collection of the data: Mahajan R, Singh A, Kaur K, Sood A. Analysis and interpretation of the data: Sood A. Drafting of the article: Mahajan R, Midha V, Singh A, Gupta Y, Sudhakar R, Pannu AS, Singh D, Sood A. Critical revision of the article for important intellectual content: all authors. Final approval of the article: all authors.

\section{ORCID}

Mahajan R https://orcid.org/0000-0001-6726-6151

Midha V https://orcid.org/0000-0003-0192-3969

Singh A https://orcid.org/0000-0001-7163-0454

Mehta V https://orcid.org/0000-0002-8867-9526

Sood A https://orcid.org/0000-0001-6961-6389 


\section{REFERENCES}

1. Sood A, Mahajan R, Juyal G, et al. Efficacy of fecal microbiota therapy in steroid dependent ulcerative colitis: a real world intention-to-treat analysis. Intest Res 2019;17:78-86.

2. Sood A, Mahajan R, Singh A, et al. Role of faecal microbiota transplantation for maintenance of remission in patients with ulcerative colitis: a pilot study. J Crohns Colitis 2019;13:13111317.

3. Collado MC, Rautava S, Isolauri E, Salminen S. Gut microbiota: a source of novel tools to reduce the risk of human disease? Pediatr Res 2015;77:182-188.

4. Singh S, Dulai PS, Zarrinpar A, Ramamoorthy S, Sandborn WJ. Obesity in IBD: epidemiology, pathogenesis, disease course and treatment outcomes. Nat Rev Gastroenterol Hepatol 2017; 14:110-121.

5. Harper JW, Zisman TL. Interaction of obesity and inflammatory bowel disease. World J Gastroenterol 2016;22:7868-7881.
6. Patel KV, Farrant P, Sanderson JD, Irving PM. Hair loss in patients with inflammatory bowel disease. Inflamm Bowel Dis 2013;19:1753-1763.

7. Hayashi A, Mikami Y, Miyamoto K, et al. Intestinal dysbiosis and biotin deprivation induce alopecia through overgrowth of Lactobacillus murinus in mice. Cell Rep 2017;20:1513-1524.

8. Rebello D, Wang E, Yen E, Lio PA, Kelly CR. Hair growth in two alopecia patients after fecal microbiota transplant. ACG Case Rep J 2017;4:e107.

9. Jacques P, Elewaut D. Joint expedition: linking gut inflammation to arthritis. Mucosal Immunol 2008;1:364-371.

10. Scher JU, Littman DR, Abramson SB. Microbiome in inflammatory arthritis and human rheumatic diseases. Arthritis Rheumatol 2016;68:35-45.

11. Herbst T, Sichelstiel A, Schär C, et al. Dysregulation of allergic airway inflammation in the absence of microbial colonization. Am J Respir Crit Care Med 2011;184:198-205. 